\title{
Tanshinone IIA inhibits high glucose-induced proliferation, migration and vascularization of human retinal endothelial cells
}

\author{
KESHUN FAN ${ }^{1,2}$, SUYAN LI ${ }^{2}$, GAOQIN LIU ${ }^{1}$, HONGHUA YUAN ${ }^{3}$, LI MA $^{3}$ and PEIRONG LU ${ }^{1}$ \\ ${ }^{1}$ Department of Ophthalmology, The First Affiliated Hospital of Soochow University, Suzhou, Jiangsu 215006; \\ ${ }^{2}$ Department of Ophthalmology, The First People's Hospital of Xuzhou; ${ }^{3}$ Research Facility Center \\ for Morphology of Xuzhou Medical University, Xuzhou, Jiangsu 221002, P.R. China
}

Received June 24, 2016; Accepted August 21, 2017

DOI: $10.3892 / \mathrm{mmr} .2017 .7743$

\begin{abstract}
Diabetic retinopathy is the most universal and severe complication of diabetes mellitus. The primary aim of the present study was to determine whether tanshinone IIA (TSA) has an inhibitory effect on the proliferation, migration and vascularization of human retinal endothelial cells (HREC) under high glucose (HG) conditions and the associated underlying mechanism. It was demonstrated that TSA exhibited a significant inhibitory effect on the proliferation, migration and vascularization of HRECs in a dose-dependent manner, under conditions of high glucose $(25 \mathrm{mM})$ medium. However, there was no distinct inhibitory effect on HREC proliferation, migration and vascularization under normal glucose (NG, $5.5 \mathrm{mM}$ glucose) conditions. Reverse transcription-quantitative polymerase chain reaction, western blotting and immunofluorescence experiments were conducted to evaluate the effects of TSA on the expression levels of vascular endothelial growth factor (VEGF) and intercellular adhesion molecule (ICAM)-1. It was demonstrated that TSA significantly downregulated the expression levels of VEGF and ICAM-1 in a dose-dependent manner under HG conditions. Overall, the results of the present study indicate that TSA-mediated inhibition of proliferation, migration and vascularization in HRECs may be associated with its ability to affect the expression levels of VEGF and ICAM-1.
\end{abstract}

\section{Introduction}

Diabetes mellitus (DM) is a type of endocrine disease which is estimated to affect 284.6 million people worldwide. According to previous reports, the number of people with DM is predicted to increase to 438 million in 2030, which accounts for $6.4 \%$ of the global population (1). Diabetic retinopathy (DR), the

Correspondence to: Dr Peirong Lu, Department of Ophthalmology, The First Affiliated Hospital of Soochow University, 188 Shizi Street, Suzhou, Jiangsu 215006, P.R. China

E-mail: lupeirong@suda.edu.cn

Key words: tanshinone IIA, diabetic retinopathy, diabetes mellitus most universal and severe complication of DM, is one of the primary causes of blindness in adults (2). The occurrence of DR is associated with the duration of DM. Retinopathy rarely occurs in the first few years of diabetes, however the likelihood of development increases to 50\% following 10 years, and $90 \%$ following 25 years of suffering with DM (3). DR results in loss of vision and blindness, which may reduce quality of life and result in an economic burden to patients and the country. Therefore, the prevention and treatment of DR is of primary concern for researchers and clinicians.

The pathogenesis of DR has not been completely elucidated, however it is believed to be correlated with synergistic effects of a variety of factors. The pro-angiogenic cytokine vascular endothelial growth factor (VEGF) is the primary factor involved in neovascularization, which is the pathological basis of DR (4-7). VEGF upregulation has been detected in the vitreous humour and the fibrovascular tissues from eyes with DR (8-13). VEGF activates two tyrosine kinase receptors, VEGF receptor (R)-1 and VEGFR-2. These two receptors regulate the physiological and pathological angiogenesis process. It has been demonstrated that VEGFR-2 activation stimulates endothelial cell proliferation, migration, and survival, in addition to mediating angiogenesis and microvascular permeability in DR (14). Furthermore, various studies suggest that leukocyte aggregation resulting from overexpression of intercellular adhesion molecule (ICAM)-1 is an important factor in inducing the destruction of the blood-retinal barrier (15). Previous studies indicate that leukocyte adhesion is important in the pathogenesis of DR. It is reported that the region of endothelial cell destruction, capillary loss, and leukocyte extravasation is often adjacent to static leukocytes (16). Therefore, evaluation of VEGF and ICAM-1 expression levels is commonly used to assess retinal vascular injury in DM.

Salvia miltiorrhiza Bunge (Danshen), which is an important source of numerous active natural products, is divided into aqueous and lipid soluble (diterpene) fractions (17). Tanshinone IIA (TSA) is the most active diterpenoid quinine pigment in Danshen. The prominent benefits of TSA on DM have been validated in numerous studies. It is reported that TSA ameliorates glucose tolerance and decreases the low-density to high-density lipoprotein ratio without altering food intake in a high-fat diet induced obese animal model (18). Similar results have been reported in $\mathrm{db} / \mathrm{db}$ mice with DM, whereby TSA 
reduces the level of blood glucose (19). Furthermore, treatment with TSA reduces infarct area and ameliorates cardiac dysfunction following ischemia/reperfusion injury in diabetic rats (20). TSA has additionally been demonstrated to inhibit vascular smooth muscle cell proliferation and alleviate intimal hyperplasia (21). However, to the best of the author's knowledge, no studies to date have focused on the effect of TSA on HRECs under high glucose (HG) conditions mimicking DM.

The present study investigated the effects of TSA on the proliferation, migration and vascularization of HRECs under HG conditions. Following this, the effects of TSA on VEGF and ICAM-1 expression levels in HREC were analyzed.

\section{Materials and methods}

Reagents. TSA was obtained from Sigma-Aldrich (Merck KGaA, Darmstadt, Germany). ICAM-1 and VEGF antibodies were purchased from Abcam (Cambridge, UK). Antibodies for $\beta$-actin was purchased from Cell Signaling Technology Inc., (Danvers, MA, USA).

Cell culture. The HREC cell line was purchased from Shanghai Cell bank, Type Culture Collection Committee, Chinese Academy of Sciences (Shanghai, China). The cells were cultured in Dulbecco's modified Eagle's medium (DMEM) containing normal glucose (NG, $5.5 \mathrm{mM})$ or $\mathrm{HG}(25 \mathrm{mM})$, from Invitrogen; Thermo Fisher Scientific Inc., (Waltham, MA, USA), supplemented with $10 \%$ fetal bovine serum (Transgen Biotech, Beijing, China, http://www.transgen.com.cn/) and grown in a humidified incubator at $37^{\circ} \mathrm{C}$, in an environment containing $5 \% \mathrm{CO}_{2}$.

Cell growth assay. Cell viability was measured with a Cell Counting Kit-8 (CCK-8; Dojindo Molecular Technologies, Inc., Kumamoto, Japan). The single cell suspension $\left(5 \times 10^{4} / \mathrm{ml}, 100 \mu \mathrm{l}\right)$ of HRECs were dispensed in a 96-well plate and cultured for $0,24,48$ and $72 \mathrm{~h}$. At the designated time point, $10 \mu \mathrm{l}$ of CCK- 8 reagent was added into each well and incubated for another $1.5 \mathrm{~h}$. Then, the absorbance was measured at a wavelength of $450 \mathrm{~nm}$, using a scanning microplate reader.

Wound healing assay. The migratory behavior of cells was assessed using a wound healing assay as previously described (22). Briefly, a monolayer of HRECs were wounded with a plastic pipette tip and rinsed twice with PBS to remove the dead cells and incubated in serum-free medium. At the designated time-point $(0,24$ and $48 \mathrm{~h})$, five randomly selected fields were photographed under an Olympus IX-71 inverted microscope.

Angiogenesis in vitro. Matrigel (BD Biosciences, Franklin Lakes, NJ, USA) was added into an eight-chamber slide and allowed to gel for $2 \mathrm{~h}$ at $37^{\circ} \mathrm{C}$. Cells were serum deprived overnight in serum-free medium prior to detaching. The cells $\left(5 \times 10^{4}\right)$ were suspended in the NG or HG medium containing $0,10,20,30 \mu \mathrm{g} / \mathrm{ml}$ TSA and were added to each chamber. Cell migration and rearrangement were recorded following $6 \mathrm{~h}$. Randomly selected fields were photographed using an Olympus IX-71 inverted microscope.
RNA extraction, $c D N A$ synthesis and reverse transcription-quantitative polymerase chain reaction ( $R T-q P C R)$. RT-qPCR was performed as previously described (23). RNA was isolated from the cells treated with $\mathrm{NG}$ medium or $\mathrm{HG}$ medium supplemented with $0,10,20$ or $30 \mu \mathrm{g} / \mathrm{ml}$ TSA and the cDNA was constructed with the M-MLV reverse transcription reagents (Roche Diagnostics, Basel, Switzerland) according to the manufacturer's protocol. RT-qPCR was carried out using an ABI 7300 real-time PCR instrument (Applied Biosystems; Thermo Fisher Scientific, Inc.) using SYBR Green (Roche Diagnostics, Basel, Switzerland). The thermocycling conditions were: $5 \mathrm{~min}$ at $95^{\circ} \mathrm{C}$ for pre-denaturation, $30 \mathrm{sec}$ at $95^{\circ} \mathrm{C}$, $30 \mathrm{sec}$ at $55^{\circ} \mathrm{C}, 30 \mathrm{sec}$ at $72^{\circ} \mathrm{C}$, the three steps $\left(30 \mathrm{sec}\right.$ at $95^{\circ} \mathrm{C}$, $30 \mathrm{sec}$ at $55^{\circ} \mathrm{C}$ and $30 \mathrm{sec}$ at $72^{\circ} \mathrm{C}$ ) were repeated for 25 cycles and the final step was $5 \mathrm{~min}$ at $72^{\circ} \mathrm{C}$. The primers used to amplify VEGF and $\beta$-actin were as follows: Forward, TGG TCCCAGGCTGCACCCAT and reverse, CGCATCGCATCA GGGGCACA for VEGF; forward, CATGTACGTTGCTAT CCAGGC and reverse, CGCTCGGTGAGGATCTTCATG for $\beta$-actin. The products were $184 \mathrm{bp}$ and $195 \mathrm{bp}$, respectively. For each sample, the threshold cycle $(\mathrm{Ct})$ was determined and normalized to the average of the housekeeping gene $(\Delta \mathrm{Ct}=\mathrm{Ct}$ unknow $\mathrm{n}$ - Ct housekeeping gene). The gene transcript levels in each sample was determined using the $2^{-\Delta \Delta \mathrm{Cq}}$ method (24).

Protein extraction and western blotting. At the designated time points, the cells were harvested and lysed with radioimmunoprecipitation assay buffer (Beyotime Institute of Biotechnology, Haimen, China). The concentrations of total protein were determined by a BCA Protein Assay kit (Beyotime Institute of Biotechnology). A total of $50 \mu \mathrm{g}$ total protein was separated by $10 \%$ SDS-PAGE and then electrophoretically transferred to a polyvinylidene fluoride membrane (EMD Millipore, Billerica, MA, USA). The membranes were incubated in 3\% bovine serum albumin (Sigma-Aldrich; Merck KGaA) in PBS for $2 \mathrm{~h}$ at room temperature, and then with primary antibodies (anti-ICAM1 antibody, cat no. ab53013, 1:500; anti-VEGFA antibody, cat no. ab183100, 1:1,000; $\beta$-actin, cat no. 3700, $1: 4,000)$ at $4^{\circ} \mathrm{C}$ overnight. On the following day, the membranes were incubated with secondary antibodies (goat anti-mouse IgG-HRP, 1:5,000; cat no. AP124P; goat anti-rabbit IgG-HRP, 1:5,000, cat no. AP132P) (both from EMD Millipore) at room temperature for $1 \mathrm{~h}$. Finally, the membranes were detected using Pierce ECL Plus western blotting substrate (Thermo Fisher Scientific, Inc.) and exposed to X-ray films. Band densities were quantified by ImageJ software (version 2.1.4.7; Wayne Rasband; National Institutes of Health, Bethesda, MD, USA). The relative amount of protein was determined by normalizing the densitometry value of the protein of interest to that of the loading control.

Immunofluorescence analysis. The cells grown on 13-mm diameter coverslips were treated with TSA for $48 \mathrm{~h}$ and then fixed with $4 \%$ paraformaldehyde for $30 \mathrm{~min}$ at room temperature and permeabilized in $0.2 \%$ Triton X-100 for $10 \mathrm{~min}$ at room temperature. Unspecific binding was blocked via a reaction with $10 \%$ normal goat serum (Beyotime Institute of Biotechnology) for $30 \mathrm{~min}$ at room temperature. Subsequently, the cells were incubated with rabbit anti-ICAM-1 antibody (Abcam; 1:100) overnight at $4^{\circ} \mathrm{C}$. On the following day, the 
cells were incubated with Alexa Fluor 488-conjugated goat anti-rabbit IgG (Abcam, cat no. ab150077, 1:1,000) for $1 \mathrm{~h}$ at room temperature. Finally, the nuclei were stained with 4,6-diamidino-2-phenylindole (DAPI; $1 \mu \mathrm{g} / \mathrm{ml}$; Sigma-Aldrich; Merck KGaA) for $10 \mathrm{~min}$ at room temperature. The cells were observed and recorded using a fluorescence microscope (IX71; Olympus Corporation, Tokyo, Japan). The fluorescence was quantified by Image J Software (version 2.1.4.7; Wayne Rasband, National Institutes of Health, MD).

Statistical analysis. The results are representative of experiments that were repeated $\geq$ three times and quantitative data were expressed as the mean \pm standard error. Statistical analyses were performed using SPSS software, version 13.0 (SPSS, Inc., Chicago, IL, USA). Differences in multiple groups were determined using a one-way analysis of variance followed by a Tukey's post-hoc test. Comparisons between two groups were performed using a Student's t-test. $\mathrm{P}<0.05$ was considered to indicate a statistically significant difference.

\section{Results}

TSA inhibits high glucose-induced HREC proliferation. In order to investigate the role of TSA on HRECs under high and normal concentrations of glucose, the present study evaluated the effects of 10,20 and $30 \mu \mathrm{g} / \mathrm{ml}$ TSA on the proliferation of HRECs under the conditions of high and normal glucose, using a CCK-8 assay. As presented in Fig. 1A, TSA exhibited a significant inhibitory effect on the proliferation of HRECs in a dose-dependent manner, under HG medium conditions. However, there was no significant inhibitory effect on the proliferation of HRECs under normal glucose conditions (Fig. 1B).

TSA suppresses high glucose-induced HREC migration. The wound-healing assay was used for evaluating the migration of HRECs under HG concentration. The wound healing was analyzed at 24 and $48 \mathrm{~h}$. It was demonstrated that high glucose stimulated the migration of HRECs, however, TSA significantly suppressed high glucose-induced HRECs migration in a dose-dependent manner (Fig. 2).

TSA inhibits high glucose-induced HREC vascularization. The in vitro matrigel angiogenesis model was performed to evaluate the vascularization of HRECs under HG concentration. As presented in Fig. 3, HG stimulated the vascularization of HRECs, however, TSA significantly inhibited HG-induced HRECs vascularization in a dose-dependent manner.

TSA downregulates VEGF and ICAM-1 expression levels in HRECs. It has been demonstrated that VEGF is important in diabetic microvascular complications by promoting retinal angiogenesis and increasing vascular permeability (25). At the same time, leukocyte aggregation that results from overexpression of ICAM-1 is an important factor in the destruction of the blood-retinal barrier, loss of retinal vascular perfusion and initiation of angiogenesis (15). The present study therefore analyzed the expression levels of VEGF and ICAM-1 using RT-qPCR, western blotting and immunofluorescence. The RT-qPCR and western blotting results indicated that TSA
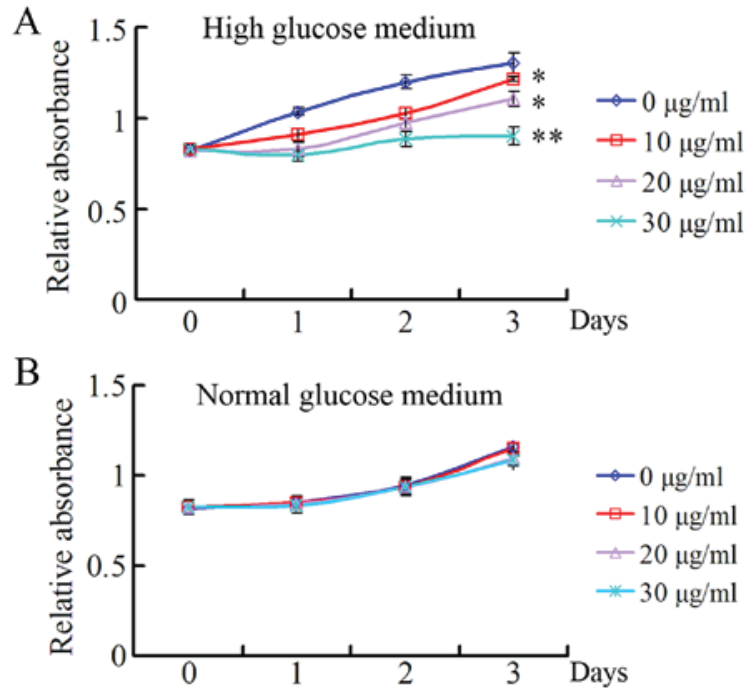

Figure 1. Effect of TSA on the proliferation of HRECs. The HRECs were cultured in HG or NG medium plus different concentrations of TSA for 24 48 and $72 \mathrm{~h}$. At the designated time point, cell proliferation was analyzed using a Cell Counting Kit-8 assay. Relative absorbance was detected using a scanning microplate reader at a wavelength of $450 \mathrm{~nm}$. All values represent the mean \pm standard error $(n=3)$. (A) TSA inhibited the proliferation of HRECs in a dose-dependent manner under HG medium conditions. ${ }^{*} \mathrm{P}<0.05$ vs. $0 \mu \mathrm{g} / \mathrm{ml}$ group; ${ }^{* *} \mathrm{P}<0.01$ vs. $0 \mu \mathrm{g} / \mathrm{ml}$. (B) TSA had no significant inhibitory effect on the proliferation of HRECs under NG conditions. HG, high glucose; NG, normal glucose; TSA, tanshinone IIA; HRECs, human retinal endothelial cells.

significantly downregulated the mRNA and protein expression levels of VEGF in a dose-dependent manner under HG conditions (Fig. 4). The immunofluorescence and western blotting indicated that TSA additionally significantly decreased the protein expression level of ICAM-1 in a dose-dependent manner, under HG conditions (Fig. 5).

\section{Discussion}

High glucose is considered an important risk factor in the development of DR. It results in cellular stress and injury of vascular pericytes and endothelial cells, and induces the formation of aberrant capillaries $(26,27)$. Endothelial cells are critical in regulating vascular tension, and damage of these cells is the earliest event that leads to irreversible structural abnormalities (28). In the present study, HRECs were used to simulate the pathogenesis of DR under conditions of high glucose. Similar to previous reports, HRECs in the present study were cultured in either normal $(5.5 \mathrm{mM})$ or high glucose $(25 \mathrm{mM})$ media $(28,29)$. In accordance with previous studies, the results of the present study indicated that high glucose promoted the proliferation, migration and vascularization of HRECs.

Tanshinone IIA (TSA), which is a major lipophilic component isolated from Danshen, demonstrates various therapeutic and pharmacological effects including vasodilative, antithrombotic, anti-inflammatory, anti-oxidant, anti-ischemic, anti-arrhythmia, anti-hyperplasia, anti-atherosclerosis and lipid-lowering properties (30). However, whether TSA ameliorates DR is still unknown. The results of the present study indicated that TSA significantly inhibited the high 


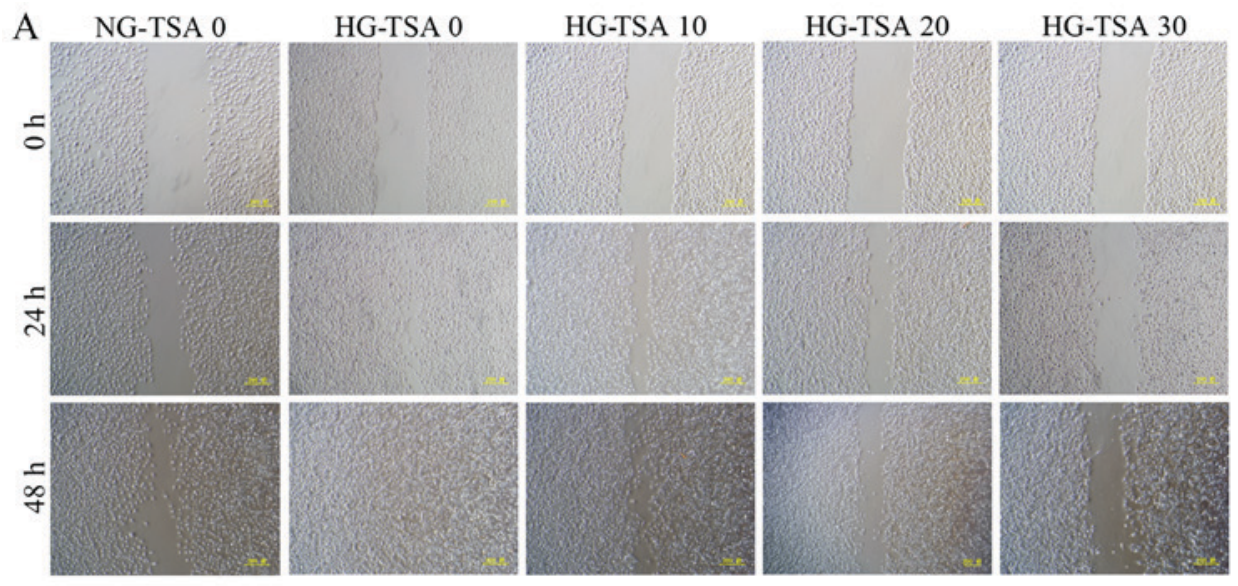

$\mathrm{B}$

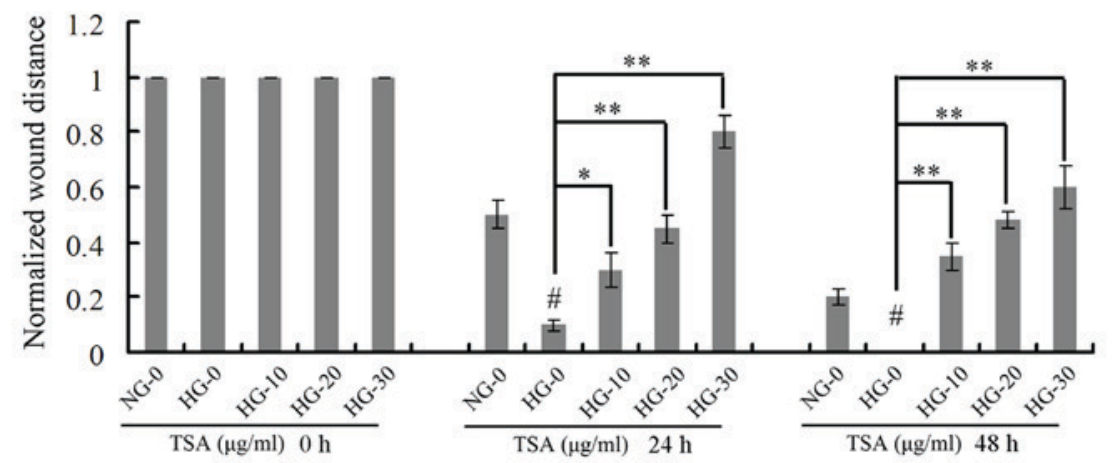

C

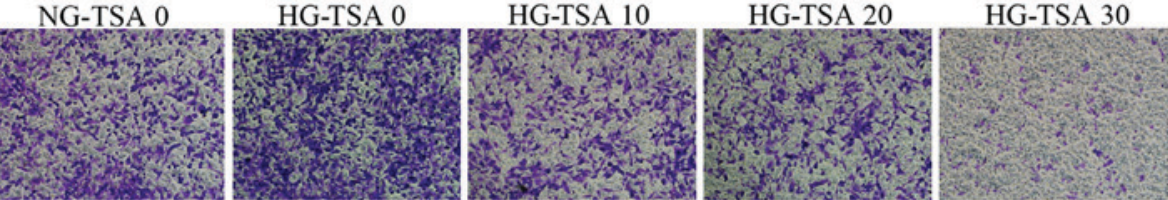

$\mathrm{D}$

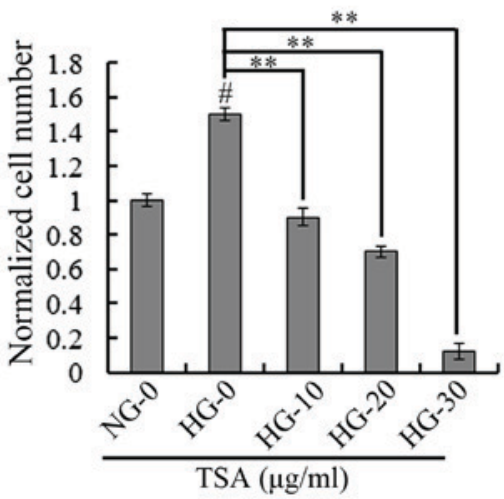

Figure 2. Effect of TSA on the migration of HRECs. A monolayer of HRECs were wounded with a plastic pipette tip and then cultured in NG or HG medium plus different concentrations of TSA for 24 and $48 \mathrm{~h}$. At the designated time $(0,24$ and $48 \mathrm{~h})$, the wound healing and transwell assays were analyzed to evaluate the effects of 10,20 and $30 \mu \mathrm{g} / \mathrm{ml}$ TSA on the migration of HRECs under HG concentrations. (A) Representative images and (B) quantification of endothelial cell migration by wound healing assay at $24 \mathrm{~h}$ and $48 \mathrm{~h}$. (C) Representative images and (D) quantification of endothelial cell migration by transwell assay. "P<0.05 vs. HG-0 group; " $\mathrm{P}<0.01$ vs. HG-0 group; ${ }^{*} \mathrm{P}<0.01$ vs. NG-0 group. HG, high glucose; NG, normal glucose; TSA, tanshinone IIA; HRECs, human retinal endothelial cells.

glucose induced proliferation, migration and vascularization of HRECs. Based on these findings, it was hypothesized that TSA may act as an alternative inhibitor for intervention of DR.

The angiogenic cascade is a complicated and multi-step process. The migration and proliferation of vascular endothelial cells are the primary step in angiogenesis, and following this, endothelial cells differentiate into a capillary-like network (31). This process is tightly controlled by a battery of pro- and anti-angiogenic factors under physiological conditions. An imbalance of these factors may result in severe pathological consequences. (32). Notably, VEGF is regarded as a primary factor of the aberrant angiogenesis in response to high glucose. Therefore, anti-VEGF therapy is considered a sufficient treatment strategy for DR $(33,34)$. In 2012, ranibizumab, a monoclonal antibody targeting VEGF designed for use in the eye, became the first and only U.S. Food and Drug Administration-approved reagent for DR $(34,35)$. In the present study, the results of mRNA and protein analysis revealed 

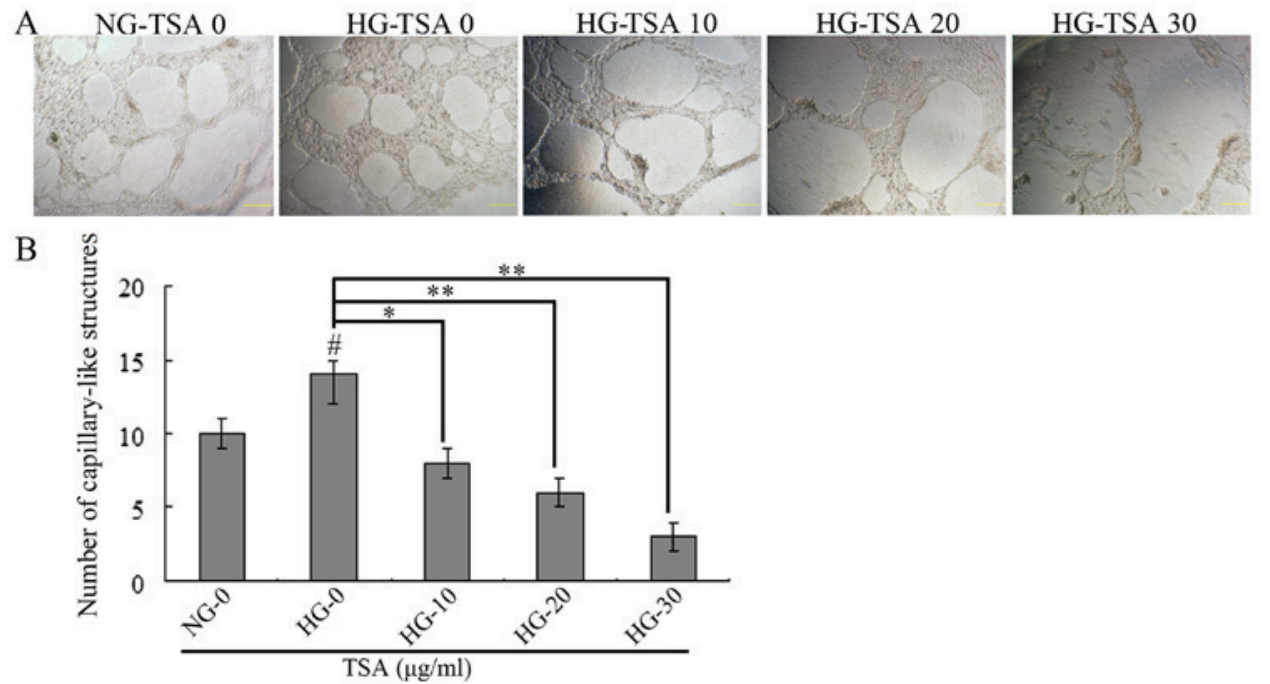

Figure 3. Effect of TSA on the vascularization of HRECs. The HRECs were suspended in the NG or HG medium containing $0,10,20,30 \mu \mathrm{g} / \mathrm{ml}$ TSA and were cultured on the matrigel containing chamber to each chamber. (A) Representative images and (B) quantification of endothelial cell migration and rearrangement. Images reveal effects on vascularization of HRECs under HG medium following $6 \mathrm{~h}$ culturing with 10,20 and $30 \mu \mathrm{g} / \mathrm{ml}$ TSA. ${ }^{*} \mathrm{P}<0.05$ vs. HG-0 group; ${ }^{* *} \mathrm{P}<0.01$ vs. HG-0 group; ${ }^{*} \mathrm{P}<0.01$ vs. NG-0 group. HG, high glucose; NG, normal glucose; TSA, tanshinone IIA; HRECs, human retinal endothelial cells.

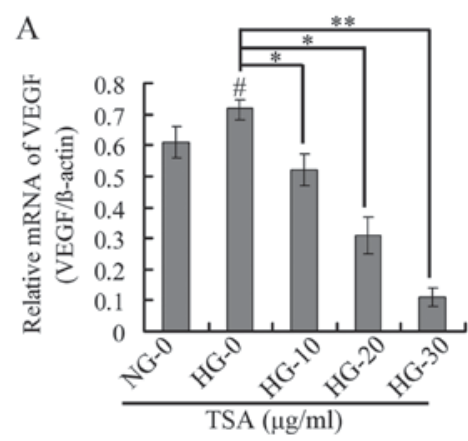

B
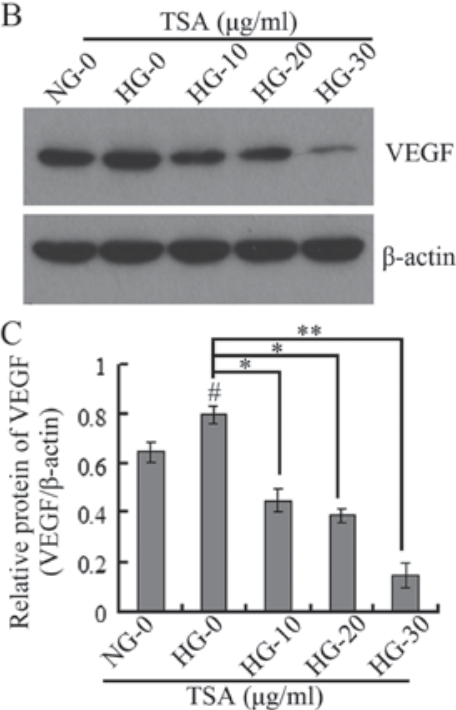

Figure 4. Effect of TSA on the expression of VEGF. Following HREC treatment with different concentrations of TSA for $48 \mathrm{~h}, \mathrm{RT}$-qPCR and western blotting were performed to assess the mRNA and protein expression levels of VEGF. (A) RT-qPCR revealed that TSA significantly downregulated the mRNA of VEGF in a dose-dependent manner, under HG conditions. (B) Representative image and (C) quantification of western blotting demonstrated that TSA significantly downregulated the protein expression level of VEGF in a dose-dependent manner under HG conditions. ${ }^{~} \mathrm{P}<0.05$ vs. NG- 0 group; ${ }^{*} \mathrm{P}<0.05$ and ${ }^{* *} \mathrm{P}<0.01$ vs. HG-0 group. RT-qPCR, reverse transcription-quantitative polymerase chain reaction; VEGF, vascular endothelial growth factor; HG, high glucose; NG, normal glucose; TSA, tanshinone IIA; HRECs, human retinal endothelial cells.

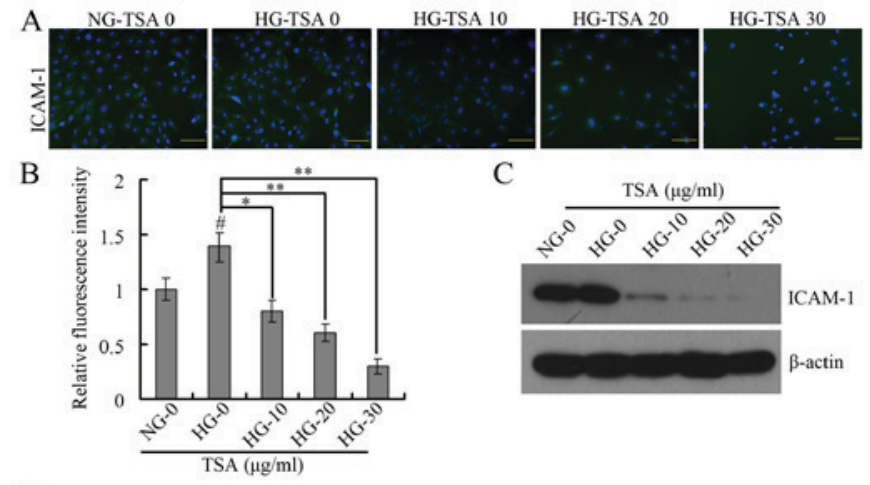

$\mathrm{D}$

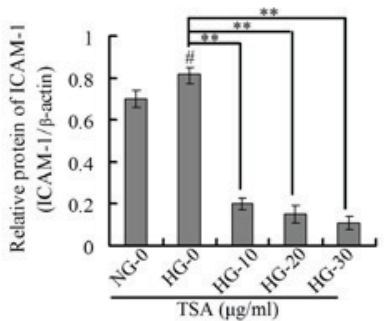

Figure 5. Effect of TSA on the expression of ICAM-1. Following HREC treatment with different concentrations of TSA for $48 \mathrm{~h}$, immunofluorescence and western blotting were performed to assess the protein expression level of ICAM-1. (A) Representative image and (B) quantification of immunofluorescence staining revealed that TSA significantly downregulated the protein expression level of ICAM-1 in a dose-dependent manner, under HG conditions. (C) Representative image and (D) quantification of Western blotting demonstrated that TSA significantly downregulated the ICAM-1 protein expression level in a dose-dependent manner, under HG conditions. ${ }^{~} \mathrm{P}<0.05$ vs. NG-0 group; ${ }^{*} \mathrm{P}<0.05$ and $^{* * *} \mathrm{P}<0.01$ vs. HG-0 group. ICAM-1, intercellular adhesion molecule; HG, high glucose; NG, normal glucose; TSA, tanshinone IIA; HRECs, human retinal endothelial cells.

that TSA decreased the increased VEGF expression in high glucose treated HRECs. These results suggest that TSA may serve as a potential anti-VEGF reagent in the treatment of DR.

It has been reported that ICAM-1 is a primary factor of leukocyte aggregation (15). In the present study, the 
immunofluorescence and western blotting assays demonstrated that TSA decreased the increased ICAM-1 expression in high glucose treated HRECs. TSA may therefore additionally serve as an anti-ICAM-1 reagent in the treatment of DR.

In conclusion, the present study demonstrated that TSA inhibited the proliferation, migration and vascularization of HRECs by affecting the expression of VEGF and ICAM-1. Although the findings provide evidence that TSA may act as a prospective drug to restrain the development and progression of DR in the future, further in vivo studies are still required to evaluate its efficacy and safety.

\section{Acknowledgements}

The present study was supported by the National Natural Science Foundation in China (NSFC, grant no. 30972712) and Suzhou Municipal Natural Science Foundation (grant no. SYS201448).

\section{References}

1. Ginter E and Simko V: Global prevalence and future of diabetes mellitus. Adv Exp Med Biol 771: 35-41, 2012.

2. Willard AL and Herman IM: Vascular complications and diabetes: Current therapies and future challenges. J Ophthalmol 2012 209538, 2012.

3. Kowluru RA and Chan PS: Oxidative stress and diabetic retinopathy. Exp Diabetes Res 2007: 43603, 2007.

4. Witmer AN, Vrensen GF, Van Noorden CJ and Schlingemann RO: Vascular endothelial growth factors and angiogenesis in eye disease. Prog Retin Eye Res 22: 1-29, 2003.

5. Abu El-Asrar AM, Nawaz MI, Kangave D, Mairaj Siddiquei M and Geboes K: Angiogenic and vasculogenic factors in the vitreous from patients with proliferative diabetic retinopathy. J Diabetes Res 2013: 539658, 2013.

6. Kalka C, Masuda H, Takahashi T, Gordon R, Tepper O, Gravereaux E, Pieczek A, Iwaguro H, Hayashi SI, Isner JM and Asahara T: Vascular endothelial growth factor(165) gene transfer augments circulating endothelial progenitor cells in human subjects. Circ Res 86: 1198-1202, 2000.

7. Li B, Sharpe EE, Maupin AB, Teleron AA, Pyle AL, Carmeliet P and Young PP: VEGF and PIGF promote adult vasculogenesis by enhancing EPC recruitment and vessel formation at the site of tumor neovascularization. FASEB J 20: 1495-1497, 2006.

8. Aiello LP, Avery RL, Arrigg PG, Keyt BA, Jampel HD, Shah ST, Pasquale LR, Thieme H, Iwamoto MA, Park JE, et al: Vascular endothelial growth factor in ocular fluid of patients with diabetic retinopathy and other retinal disorders. N Engl J Med 331: 1480-1487, 1994

9. Wang $\mathrm{X}$, Wang $\mathrm{G}$ and Wang Y: Intravitreous vascular endothelial growth factor and hypoxia-inducible factor 1a in patients with proliferative diabetic retinopathy. Am J Ophthalmol 148 : 883-889, 2009.

10. Matsuoka M, Ogata N, Minamino K and Matsumura M: Expression of pigment epithelium-derived factor and vascular endothelial growth factor in fibrovascular membranes from patients with proliferative diabetic retinopathy. Jpn J Ophthalmol 50: 116-120, 2006.

11. Abu El-Asrar AM, Missotten L and Geboes K: Expression of hypoxia-inducible factor-1alpha and the protein products of its target genes in diabetic fibrovascular epiretinal membranes. Br J Ophthalmol 91: 822-826, 2007.

12. Lim JI,Spee Cand Hinton DR: A comparison of hypoxia-inducible factor- $\alpha$ in surgically excised neovascular membranes of patients with diabetes compared with idiopathic epiretinal membranes in nondiabetic patients. Retina 30: 1472-1478, 2010.

13. Chung EJ, Kang SJ, Koo JS, Choi YJ, Grossniklaus HE and Koh HJ: Effect of intravitreal bevacizumab on vascular endothelial growth factor expression in patients with proliferative diabetic retinopathy. Yonsei Med J 52: 151-157, 2011.

14. Shibuya M: Differential roles of vascular endothelial growth factor receptor-1 and receptor-2 in angiogenesis. J Biochem Mol Biol 39: 469-478, 2006.
15. Hubbard AK and Rothlein R: Intercellular adhesion molecule-1 (ICAM-1) expression and cell signaling cascades. Free Radic Biol Med 28: 1379-1386, 2000.

16. Barouch FC, Miyamoto K, Allport JR, Fujita K, Bursell SE, Aiello LP, Luscinskas FW and Adamis AP: Integrin-mediated neutrophil adhesion and retinal leukostasis in diabetes. Invest Ophthalmol Vis Sci 41: 1153-1158, 2000.

17. Zhou L, Zuo Z and Chow MS: Danshen: An overview of its chemistry, pharmacology, pharmacokinetics, and clinical use. J Clin Pharmacol 45: 1345-1359, 2005.

18. Gong Z, Huang C, Sheng X, Zhang Y, Li Q, Wang MW, Peng L and Zang YQ: The role of tanshinone IIA in the treatment of obesity through peroxisome proliferator-activated receptor gamma antagonism. Endocrinology 150: 104-113, 2009.

19. Hwang SL, Yang JH, Jeong YT, Kim YD, Li X, Lu Y, Chang YC, Son KH and Chang HW: Tanshinone IIA improves endoplasmic reticulum stress-induced insulin resistance through AMP-activated protein kinase. Biochem Biophys Res Commun 430: 1246-1252, 2013.

20. Zhang Y, Wei L, Sun D, Cao F, Gao H, Zhao L, Du J, Li Y and Wang H: Tanshinone IIA pretreatment protects myocardium against ischaemia/reperfusion injury through the phosphatidylinositol 3-kinase/Akt-dependent pathway in diabetic rats. Diabetes Obes Metab 12: 316-322, 2010

21. Li X, Du JR, Yu Y, Bai B and Zheng XY: Tanshinone IIA inhibits smooth muscle proliferation and intimal hyperplasia in the rat carotid balloon-injured model through inhibition of MAPK signaling pathway. J Ethnopharmacol 129: 273-279, 2010.

22. Chen Z, Liu G, Xiao Y and Lu P: Adrenomedullin22-52 suppresses high-glucose-induced migration, proliferation, and tube formation of human retinal endothelial cells. Mol Vis 20: 259-269, 2014.

23. Dai C, Liu G, Li L, Xiao Y, Zhang X and Lu P: ADP-ribosylation factor as a novel target for corneal neovascularization regression. Mol Vis 18: 2947-2953, 2012.

24. Livak KJ and Schmittgen TD: Analysis of relative gene expression data using real-time quantitative PCR and the 2(-Delta Delta C(T)) method. Methods 25: 402-408, 2001.

25. Rajah TT and Grammas P: VEGF and VEGF receptor levels in retinal and brain-derived endothelial cells. Biochem Biophys Res Commun 293: 710-713, 2002.

26. Stitt AW, McGoldrick C, Rice-McCaldin A, McCance DR, Glenn JV, Hsu DK, Liu FT, Thorpe SR and Gardiner TA: Impaired retinal angiogenesis in diabetes: Role of advanced glycation end products and galectin-3. Diabetes 54: 785-794, 2005.

27. Dagher Z, Park YS, Asnaghi V, Hoehn T, Gerhardinger C and Lorenzi M: Studies of rat and human retinas predict a role for the polyol pathway in human diabetic retinopathy. Diabetes 53: 2404-2411, 2004

28. Yuan L, Hu J, Luo Y, Liu Q, Li T, Parish CR, Freeman C, Zhu X, Ma W, Hu X, et al: Upregulation of heparanase in high-glucose-treated endothelial cells promotes endothelial cell migration and proliferation and correlates with Akt and extracellular-signal-regulated kinase phosphorylation. Mol Vis 18: 1684-1695, 2012.

29. Premanand C, Rema M, Sameer MZ, Sujatha M and Balasubramanyam M: Effect of curcumin on proliferation of human retinal endothelial cells under in vitro conditions. Invest Ophthalmol Vis Sci 47: 2179-2184, 2006.

30. Shang Q, Xu H and Huang L: Tanshinone IIA: A promising natural cardioprotective agent. Evid Based Complement Alternat Med 2012: 716459, 2012.

31. Jeganathan VS: Anti-angiogenesis drugs in diabetic retinopathy. Curr Pharm Biotechnol 12: 369-372, 2011.

32. Kumar B, Gupta SK, Saxena R and Srivastava S: Current trends in the pharmacotherapy of diabetic retinopathy. J Postgrad Med 58: 132-139, 2012.

33. Zechmeister-Koss I and Huic M: Vascular endothelial growth factor inhibitors (anti-VEGF) in the management of diabetic macular oedema: A systematic review. Br J Ophthalmol 96: 167-178, 2012.

34. Titchenell PM and Antonetti DA: Using the past to inform the future: Anti-VEGF therapy as a road map to develop novel therapies for diabetic retinopathy. Diabetes 62: 1808-1815, 2013.

35. Stewart MW: Critical appraisal of ranibizumab in the treatment of diabetic macular edema. Clin Ophthalmol 7: 1257-1267, 2013. 